\title{
Three-dimensional dynamic MR-hysterosalpingography; a new, low invasive, radiation-free and less painful radiological approach to female infertility
}

\author{
M.Unterweger ${ }^{1}$, C.De Geyter ${ }^{2}$, J.M.Fröhlich ${ }^{1}$, G.Bongartz ${ }^{1}$ and W.Wiesner ${ }^{1,3}$ \\ ${ }^{1}$ Institute of Diagnostic Radiology, Department of Radiology, University Hospital and ${ }^{2}$ Division of Gynecological Endocrinology and \\ Reproductive Medicine, University Women's Hospital, Basel, Switzerland \\ ${ }^{3}$ To whom correspondence should be addressed at: Institute of Diagnostic Radiology, University Hospital Basel, Petersgraben 4, 4031 \\ Basel, Switzerland. E-mail: wwiesner@uhbs.ch
}

BACKGROUND: The purpose of this study was to propose a new method for imaging the uterine cavity and Fallopian tube patency by three-dimensional dynamic magnetic resonance hysterosalpingography (3D dMR-HSG) and to analyse if, by using a higher viscosity contrast solution, direct visualization of the Fallopian tubes may be achieved by this new technique. METHODS: 10 consecutive infertile women underwent 3D dMR-HSG and conventional HSG as gold standard. 3D dMR-HSG consisted of injection of $20 \mathrm{ml}$ of a gadolinium-polyvidone solution into the uterine cavity while acquiring five consecutive three-dimensional (3D) T1-weighted MR-sequences. RESULTS: In three patients the catheter became dislodged during 3D dMR-HSG. However, in one of these patients the examination was still partially diagnostic. Imaging findings of 3D dMR-HSG showed good correlation with conventional hysterosalpingography and allowed 3D imaging of the uterine cavity and of Fallopian tube patency in 8/10 patients and direct visualization of the Fallopian tubes in 5/7 patients. CONCLUSION: 3D dMR-HSG represents a new and promising imaging approach to female infertility causing less pain and avoiding exposure of the ovaries to ionizing radiation. By using a higher viscosity MR-contrast agent it allows not only visualization of uterine cavity and Fallopian tube patency but also direct visualization of Fallopian tubes.

Key words: hysterosalpingography/infertility/magnetic resonance imaging

\section{Introduction}

Although it can be shown that ultrasound with saline solution is the best method to study the uterine cavity and although contrast enhanced hystero-salpingo-sonography allows visualization of the uterine cavity as well as Fallopian tube patency, conventional (X-ray) hysterosalpingography (HSG) remains the most commonly used procedure for imaging the uterine cavity, the Fallopian tubes and Fallopian tube patency in the evaluation of female infertility (Yoder and Hall, 1991; Krysiewics, 1992; Reis et al., 1998; Boudghene et al., 2001). However, conventional HSG is painful and leads unavoidably to an application of ionizing radiation to the reproductive organs of young and potentially fertile women. Furthermore, many other causes of female infertility cannot be diagnosed by X-ray-HSG and, therefore, many of these patients not only undergo conventional HSG but additionally also transabdominal and endovaginal sonography (Philipsen, Hansen, 1981; Fayez et al., 1988; Yoder and Hall, 1991; Krysiewics, 1992; Reis et al., 1998; Boudghene et al., 2001). In the past, the leading role of magnetic resonance imaging (MRI) as an additional examination in the evaluation of female infertility has been well established since MRI proved to have a very high accuracy in detecting various pelvic, uterine and ovarian pathologies (Philipsen, Hansen, 1981; Dudiak et al., 1988; Carrington et al., 1990; Dykes et al., 1991; Mitchell, 1992; Pellerito et al., 1992; Woodward et al., 1993; Ascher et al., 1995). Furthermore it could be shown that imaging the uterine cavity and Fallopian tube patency is possible by MR-HSG but until now, direct visualization of the Fallopian tubes could not be achieved by MR-HSG (Lee et al., 1996; Frye et al., 2000; Hagspiel et al., 2000; Rouanet et al., 2000; Wiesner et al., 2001). Therefore, the purpose of our study was to analyse if, by using a special higher viscosity MR-HSG-contrast solution composed especially and exclusively for this purpose, standard pelvic MRI could be combined with MR-HSG in order to enable a one-step imaging procedure for evaluation of female infertility with the advantages of causing less pain and avoidance of exposure to ionizing radiation.

Materials and methods

After the present study had been approved by the local ethics committee, 10 consecutive infertile women (age range: 22-41years; mean age: 33.8), who presented in our Division of Gynecological 


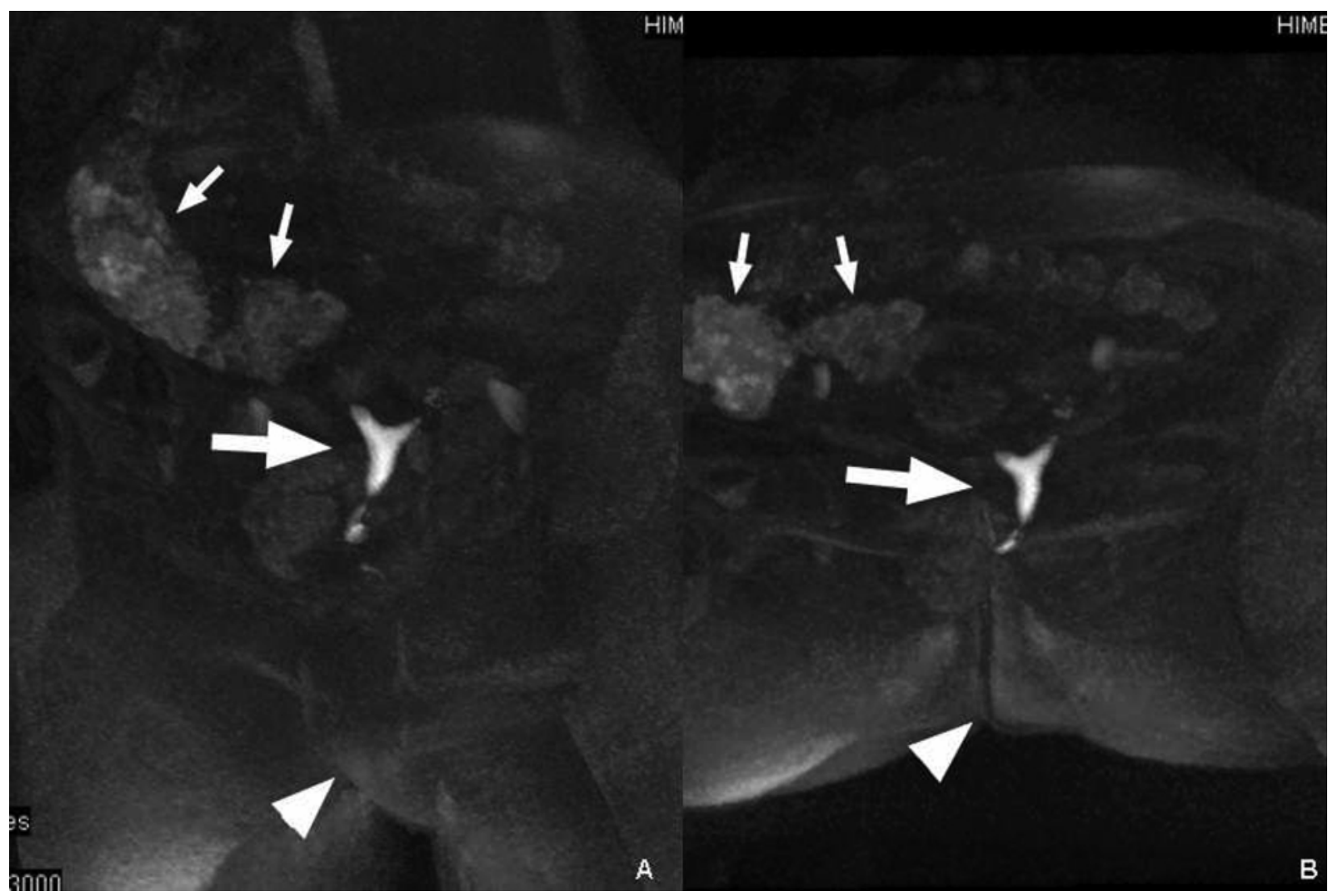

Figure 1. Three dimensional dynamic MR-HSG with overview maximum intensity projections acquired early during uterine injection of a higher viscosity gadolinium-polyvidone solution allows perfect 3D visualization of the uterine cavity within the body from different views. Left anterior oblique projection (A) and an inferior-superior projection (B). Note: the uterus is not only slightly anteflected but also mildly positioned to the left. Uterine cavity (large arrow in $\mathbf{A}$ and $\mathbf{B}$ ), colonic content (small arrows in $\mathbf{A}$ and $\mathbf{B}$ ), vulva and rima ani (arrowhead in $\mathbf{A}$ and $\mathbf{B}$, respectively).

Endocrinology and Reproductive Medicine for evaluation of female infertility and who were willing to participate, were included into our study. Written informed consent had been obtained from all patients to perform both MR-HSG and conventional HSG within 2 hours. The weight of our patients ranged from 46 to $103 \mathrm{~kg}$ (mean: 64.4). Their body height ranged from 155-176 cm (mean: 167.2).

As a first step uterine cannulation was performed by a gynaecologist using a balloon catheter (Charriere 8, Rüsch, Kernen, Germany) which was placed into the uterine cavity and blocked at its position. MR-imaging was performed in a 1.5 Tesla MR-unit (Symphony, Siemens, Erlagen). For imaging of the true pelvis standard axial and sagittal T1-w and T2-w sequences were acquired. For 3D dMR-HSG a fat-saturated 3D gradient-echo sequence was acquired with a phased array body coil ('FLASH 3D': TE/TR 3.6/1.4, Flip-angle 25, FOV $380 \mathrm{~mm}$, slice thickness $1.25 \mathrm{~mm}$, voxel size $1.4 \times 0.7 \times 1.3 \mathrm{~mm})$.

By using this three dimensional T1-weighted sequence, five consecutive data sets were acquired before, at the beginning, during early, during late, and after uterine injection of $20 \mathrm{ml}$ of a strictly germ-free gadolinium-polyvidone gel solution which was formulated exclusively for this purpose.

The solution consisted of $18.6 \mathrm{mmol} / 1$ Dotarem $^{\circledR}$ (Acidum gadoteras DCI, Guerbet, Paris, France) mixed with polyvidone (140 mg PVP C30). Dotarem ${ }^{\circledR}$, is a well known macrocyclic gadolinium-chelate complex used worldwide as a paramagnetic MR-contrast agent primarily for intravenous usage. However, since Dotarem ${ }^{\circledR}$ is an extracellular contrast agent it may also be used as a positive intestinal contrast agent for example for MR-colonographies or MR-enteroclyses and extravasation of Dotarem ${ }^{\circledR}$ into the peritoneal cavity is therefore harmless. Furthermore, Dotarem ${ }^{\circledR}$ is chemically very stable, does not bind to albumins or other proteins and does not undergo any metabolism. Therefore Dotarem ${ }^{\circledR}$ behaves intracorporally as other water-soluble and inert substances (such as mannitol or inulin) and is cleared by renal excretion within 24 hours. Polyvidone on the other hand is a viscosity increasing substance that is regularly used in conventional HSG contrast media such as Telebrix-Hystero (Guerbet, Paris France).

Viscosity of our slightly hyperosmolar contrast solution (600 mOsm $/ \mathrm{kg} \mathrm{H}_{2} \mathrm{O}$ ) could thus be increased up to $100 \mathrm{mPa} \cdot \mathrm{s}$. Each $3 \mathrm{D}$ acquisition took only 20-25 s. All patients underwent X-ray-HSG as a gold-standard immediately following 3D dMR-HSG and both examinations were performed by the same gynaecologist and by the same radiologist. Finally all patients (who did not receive any anaesthesia or analgesia for HSG) were interrogated regarding their experiences during both procedures.

\section{Results}

Standard T1-w and T2-w images were used to exclude anatomical malformations, fibroids, endometriosis, tumours, cysts or other pathologies of the genital tract as is usually carried out in daily practice. This was possible in all patients. Positioning of the catheter was always feasible. However, in three patients the catheter slipped back into the vagina during the examination. While in two of these patients this happened at the beginning of our examination resulting in a non-diagnostic MR-HSG, in one patient it happened at the end of the examination and MR-HSG was still diagnostic regarding 


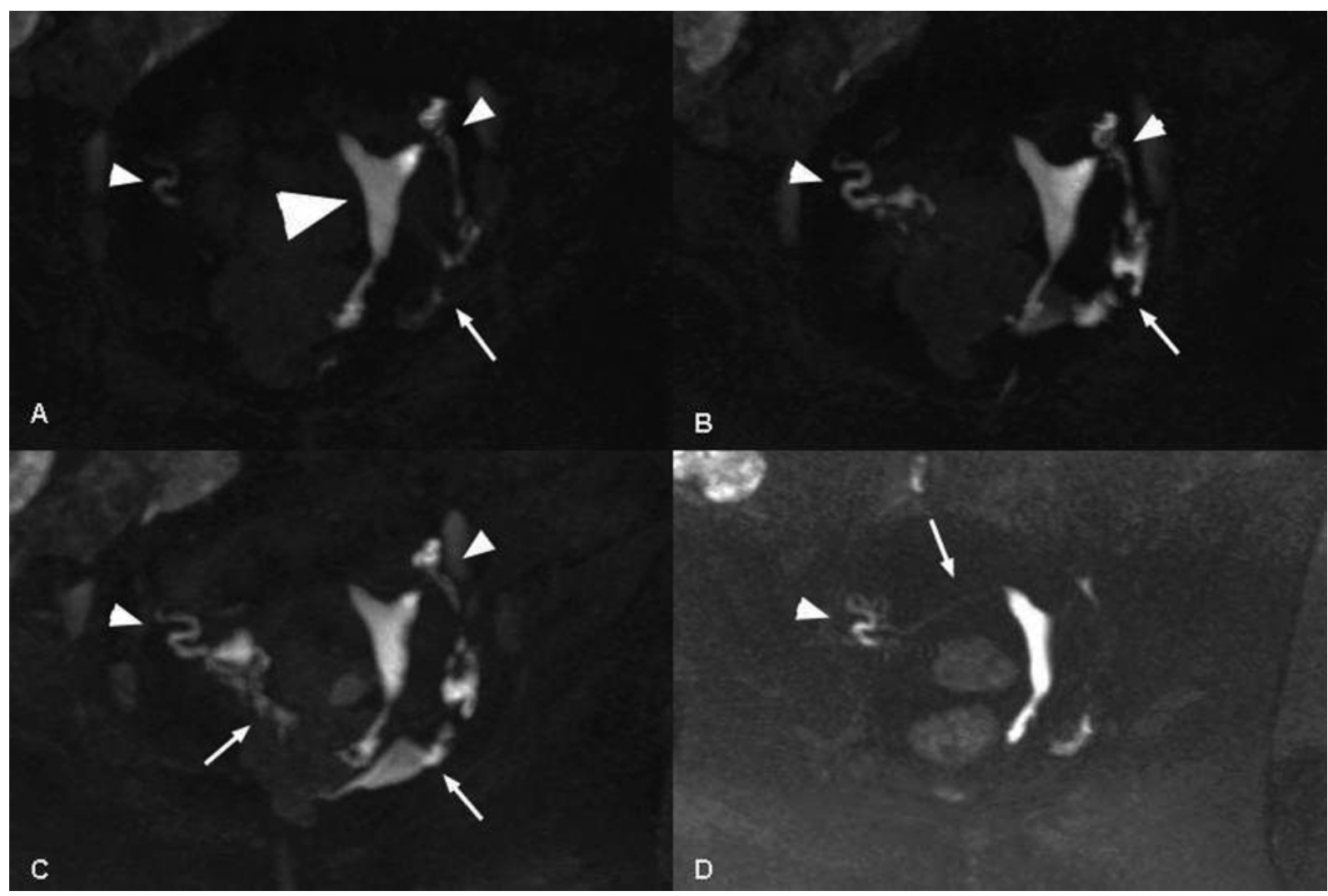

Figure 2. Three dimensional dynamic MR-HSG with maximum intensity projections (MIP) acquired early (A) and during late (B) as well as after $(\mathbf{C})$ uterine injection of a gadolinium-polyvidone solution allows perfect 3D visualization of the uterine cavity (large arrowhead in $\mathbf{A}$ ), and of the ampullary parts of the tubes (small arrowheads in $\mathbf{A}, \mathbf{B}, \mathbf{C}$ and $\mathbf{D}$ ) as well as free contrast accumulation in the peritoneal cavity (arrows in A, B and C) proving bilateral Fallopian tube patency. Multiplanar thin slice MIP-reconstructions (D) additionally allow visualization of the isthmic parts of the Fallopian tubes (arrow in D).

visualization of uterine cavity and demonstration of Fallopian tube patency.

The use of 3D dMR-HSG allowed 3D visualization of a normal uterine cavity in eight patients and confirmation of bilateral Fallopian tube patency in six, which correlated with findings of conventional HSG (Figures 1 and 2). In two patients MR-HSG showed a one-sided occlusion of the Fallopian tubes which was confirmed by X-ray-HSG. Furthermore, due to the higher viscosity of our contrast solution, direct visualization of the Fallopian tubes was possible in five of seven patients (Figure 2).

One patient with catheter dislocation felt mild pain when the catheter slipped back into her vagina but the remaining 9/10 patients stated that MR-HSG was significantly less painful than X-ray-HSG.

\section{Discussion}

At present, MRI is the most accurate method for the noninvasive diagnosis of various pelvic pathologies (Dudiak et al., 1988; Carrington et al., 1990; Dykes et al., 1991; Mitchell 1992; Pellerito et al., 1992; Woodward et al., 1993; Ascher et al., 1995). Due to an excellent resolution, direct visualization of the reproductive organs, multiplanar imaging and avoidance of radiation, MRI is therefore used worldwide as an additional examination in the evaluation of female infertility. However, so far in the daily routine, Fallopian tube patency is diagnosed by conventional X-ray-HSG, a method which is often painful due to osmotic irritation of endometrial and peritoneal tissue by iodinated contrast agents and which also leads to an application of ionizing radiation to the genital organs of young and potentially fertile women (Yoder and Hall, 1991; Krysiewics, 1992).

Proof of Fallopian tube patency may also be achieved by laparoscopy and by contrast enhanced sonography, but laparoscopy fails to provide visualization of the uterine cavity and contrast enhanced sonography has the disadvantage of being more observer dependent and, therefore, less reproducible (Philipsen, Hansen, 1981; Fayez et al., 1988; Reis et al., 1998; Boudghene et al., 2001). Furthermore, a variety of other possible causes for female infertility cannot be diagnosed accurately by conventional HSG and sonography and, therefore, many patients who are additionally referred for pelvic MRI finally have to undergo more than one of the aforementioned examinations.

Over the past years research activities on MR-HSG were intensified but 3D and dynamic visualization of the human uterine cavity and of Fallopian tube patency, together with direct visualization of Fallopian tubes has not yet been achieved (Lee et al., 1996; Frye et al., 2000; Hagspiel et al., 2000; Rouanet et al., 2000; Wiesner et al., 2001). Therefore, combined with standard pelvic MRI our method represents a possible one-step imaging approach to female infertility.

The fact that dislocation of the catheter occurred in three 
patients was a limitation and it is possible that this happened during the transfer to the MR system, where catheter repositioning was not possible anymore due to the narrow MR tube; however using open MR systems in which catheter positioning and repositioning could be done easily within the MR unit might solve this problem.

Further research and further technical optimization is needed as well as larger blinded comparative studies to evaluate the diagnostic accuracy of this method in regard to detection of various uterine and Fallopian pathologies compared with conventional HSG, laparoscopy and sonography.

However, one can state at the moment that the advantages of less pain during the examination, of more information about all organs of the true pelvis and especially of avoidance of exposure to ionizing radiation, do compensate for the longer examination time and for the expected higher costs of this new technique, which might evolve to a well-accepted alternative to conventional HSG and probably even replace this old method in the near future.

\section{References}

Ascher, S.M., Agrawal, R., Bis, K.G., Brown, E.D. and Semelka, R.C.(1995) Endometriosis: appearance and detection with conventional and contrast enhanced fat-suppressed spin-echo techniques. J. Magn. Reson. Imaging, 5, 251-257.

Boudghene, F.P., Bazot, M., Robert, Y., Perrot, N., Rocourt, N., Antoine, J.M., Morris, H., Leroy, J.L., Uzan, S. and Bigot, J.M. (2001) Assessment of Fallopian tube patency by HyCoSy: comparison of a positive contrast agent with saline solution. Ultrasound Obstet. Gynecol., 18, 525-530.

Carrington, B.M., Hricak, H., Nuruddin, R.N., Secaf, E., Laros, R.K. Jr and Hill, E.C. (1990) Mullerian ducts anomalies: MR imaging evaluation. Radiology, 176, 715-720.

Dudiak, C.M., Turner, D.A., Patel, S.K., Archie, J.T., Silver, B. and Norusis, M. (1988) Uterine leiomyomas in the infertile patient: preoperative localisation with MR imaging versus US and hysterosalpingography. Radiology, 167, $627-630$
Dykes, T.A., Isler, R.J. and McLean, A.C. (1991) MR imaging of Asherman syndrome: total endometrial obliteration. J. Comput. Assist. Tomogr., 15, 858-860.

Fayez, J.A., Mutie, G. and Schneider, P.J. (1988) The diagnostic value of hysterosalpingography and laparoscopy in infertility investigation. Int. J. Fertil., 33, 98-101.

Frye, R.E., Ascher, S.M. and Thomasson, D. (2000) MR hysterosalpingography: protocol development and refinement for simulating normal and abnormal fallopian tube patency - feasibility study with a phantom. Radiology, 214, 107-112.

Hagspiel, K.D., Altes, T.A., Mugler, J.P., 3rd and Brookman, J.R. (2000) Magnetic resonance hysterography and hysterosalpingography using hyperpolarized (3)He: demonstration of feasibility in an animal model. J. Magn. Reson. Imaging, 12, 1009-1013.

Krysiewics, S. (1992) Infertility in women: diagnostic evaluation with hysterosalpingography and other imaging techniques. Am J. Roentgenol. 159, 253-261.

Lee, F.T. Jr, Grist, T.M., Nelson, K.G., Chosy, S.G., Rappe, A.H., Shapiro, S.S. and Kelcz, F. (1996) MR Hysterosalpingography in a rabbit model. J. Magn. Reson. Imaging, 6, 300-304.

Mitchell, D.G. (1992) Benign diseases of the uterus and ovaries. Applications of magnetic resonance imaging. Radiol. Clin. North Am., 30, 777-787.

Pellerito, J.S., McCarthy, S.M., Doyle, M.B., Glickman, M.G. and DeCherney, A.H. (1992) Diagnosis of uterine anomalies: relative accuracy of MR imaging, endovaginal sonography, and hysterosalpingography. Radiology, 183, 795-800.

Philipsen, T. and Hansen, B.B. (1981) Comparative study of hysterosalpingography and laparoscopy in infertile patients. Acta Obstet. Gynecol. Scand., 60, 149-151.

Reis, M.M., Soares, S.R., Cancado, M.L. and Camargos, A.F. (1998) Hysterosalpingo-contrast-sonography (HyCoSy) with SH U 454 (Echovist) for the assessment of tubal patency. Hum. Reprod., 13, 3049-3052.

Rouanet De Lavit, J.P., Maubon, A.J. and Thurmond, A.S. (2000) MR Hysterography performed with saline injection and fluid attenuated inversion recovery sequences: initial experience. Am. J. Roentgenol., 175, 774-776.

Wiesner, W., Ruehm, S.G., Bongartz, G., Kaim, A., Reese, E. and De Geyter, C. (2001) Three-dimensional dynamic MR-Hysterosalpingography: a preliminary report. European Radiology, 11, 1439-1444.

Woodward, P.J., Wagner, B.J. and Farley, T.E. (1993) MR imaging in the evaluation of female infertility. Radiographics, 13, 293-310.

Yoder, I.C. and Hall, D.A. (1991) Hysterosalpingography in the 1990s. Am J. Roentgenol., 157, 675-683.

Submitted on June 11, 2002; accepted on August 8, 2002 